\title{
Development of a Capillary High-performance Liquid Chromatography Tandem Mass Spectrometry System Using SWIFT Technology in an Ion Trap/Reflectron Time-of-flight Mass Spectrometer
}

\author{
Ling He, Jing-Tao Wu, Steve Parus and David M. Lubman* \\ Department of Chemistry, University of Michigan, Ann Arbor, MI 48109-1055, USA \\ SPONSOR REFEREE: G. Dollinger, Small Molecule Drug Discovery, Chiron Corporation, 4560 Horton Street, \\ Emeryville, CA 94608, USA
}

\begin{abstract}
In this work a capillary high-performance liquid chromatography/tandem mass spectrometry system has been developed in a quadrupole ion trap/reflectron time-of-flight mass spectrometer. The broadband excitation for isolating a precursor ion is achieved by generating the stored waveform inverse Fourier transform waveform in real time. Following the isolation and cooling periods, an auxiliary AC voltage (tickle) is applied to the entrance end cap of the ion trap to generate collision induced dissociation. The amplitude of the tickle voltage is optimized on-line using a feedback method which involves having two computers communicating with each other. This system has been tested with a 4-peptide mixture and tandem mass spectra were obtained for each peptide. (C) 1997 John Wiley \& Sons, Ltd.
\end{abstract}

Received 8 August 1997; Accepted 15 August 1997

Rapid. Commun. Mass Spectrom. 11, 1739-1748 (1997)

No. of Figures: 7 No. of Tables: 1 No. of Refs: 35

Electrospray ionization (ESI) mass spectrometry has become a powerful means for the on-line detection of separated mixtures in solution for proteins, peptides ${ }^{1-6}$ and small molecules. Mass spectrometric detection can differentiate mixtures not only by their retention time but also by their mass and fragmentation patterns. Due to the acidic nature of the mobile phase resulting from organic modifiers such as trifluoroacetic acid (TFA) and the existence of relatively abundant basic residues (arginine, lysine and histidine), multiply charged positive ions are typically observed. This property is especially important for generating MS/MS spectra using low energy collision induced dissociation (CID) since the multiply charged ions are more easily fragmented than singly charged ions when undergoing collisions with neutral gas molecules such as $\mathrm{He}$ and Ar. ${ }^{7}$

There are several approaches to generating MS/MS data and fragmentation. In electrospray skimmer CID the fragmentation takes place in the region between the capillary exit and the skimmer of the electrospray interface. ${ }^{8-10}$ This method can generate extensive fragmentation. However, this is not a tandem method and if several different multiply charged species are produced by the electrospray source, the MS/MS spectrum will be complicated and difficult to interpret. A more general approach, known as tandem mass spectrometry, includes selecting precursor ions prior to the CID process. The selection of the precursor ions can be achieved in two ways: tandem-in-space or tandem-in-

\footnotetext{
*Correspondence to: D. M. Lubman, Department of Chemistry, University of Michigan, Ann Arbor, MI 48109-1055, USA Contract/grant sponsor: National Institute of Health; Contract/grant number: R01GM49500

Contract/grant sponsor: National Science Foundation;

Contract/grant number: BIR-9513878
}

time methodologies. The tandem-in-space approach occurs where the parent ion selection-CID-daughter ion detection stages are spatially separated. Typical mass spectrometers of this type are triple quadrupole and tandem-sector instruments. ${ }^{11-14}$ The second approach, tandem-in-time, includes parent ion selection with CID-daughter ion detection sequentially in time but in the same region of space. Typical mass spectrometers of this type include Fourier transform mass spectrometers (FTMS) and the quadrupole ion trap. ${ }^{15-17}$

Recently the QIT has become a popular detection device for ESI-generated ions due to its high duty cycle over a broad mass range and its relatively low cost. These properties are essential for sensitive detection of ions in a continuous ion beam source such as electrospray ionization. Another important property of the ion trap is that the pressure inside is relatively high $\left(\sim 10^{-3}\right.$ Torr) for efficient trapping of externally injected ions. This makes the QIT well suited for interfacing to online methods such as high-performance liquid chromatography (HPLC). Moreover, the use of a high pressure buffer gas makes fragmentation via CID possible. When applying an auxiliary AC voltage to the endcap of the ion trap, the ions whose secular frequency match the frequency of the AC voltage will acquire energy from the waveform and become activated. The ions may undergo multiple collisions with the buffer gas molecules until they dissociate. There have been many reports of using this method for peptide sequencing. ${ }^{17,18}$

In recent work, we have developed an ion trap storage/reflectron time-of-flight (IT/reTOF) hybrid mass spectrometer in which the ions are accumulated by the ion trap, and subsequently ejected from the ion trap by applying a DC pulse to the endcap and detected 
by a reflectron TOF device. ${ }^{19}$ This device can also be used for the selective ion storage and for performance of tandem mass spectrometry. Using the non-scanning capabilities of the reTOF, the ions can be detected in a single spectrum in less than $200 \mu$ s and a duty cycle of nearly $80 \%$ can be achieved even for MS/MS anlaysis. The IT/reTOF system has been coupled to microscale separation techniques such as capillary HPLC and capillary electrophoresis (CE). ${ }^{20-22} \mathrm{LC} / \mathrm{MS} / \mathrm{MS}$ of peptides in the direct injection mode has already been reported. ${ }^{23,24}$ In order to perform on-line tandem mass spectrometry, this system must be able to detect the elution of target ions and to optimize on-line the amplitude of the AC activation voltage.

Stored waveform inverse Fourier transform (SWIFT) is a technology which was first developed to excite all the ions (broadband excitation) trapped inside a Fourier transform ion cyclotron resonance (FTICR) mass spectrometer. ${ }^{25,26}$ It is thus by far the most general means for broadband, mass-selective excitation and ejection. This technology has been recently employed in the quadrupole ion trap for performing tandem mass spectrometry for both continuous and pulsed ion sources. $^{27-29}$ The advantage of SWIFT is that by creating notches in the frequency domain magnitude spectrum, the resulting time-domain waveform will not contain frequency components which activate those ions whose secular frequency fall in the notch, a.k.a. selective ejection. This provides selective accumulation of ions inside the ion trap and significantly improves the sensitivity by elimination of the background ions that can saturate the trap. ${ }^{30-32}$ The total ion chromatogram (TIC) for an isolated ion provides the elution profile without interference from non-isobaric coeluents. The TIC information of the isolated ion can also be used for detecting the existence of the target ion. Following isolation, an auxiliary AC voltage (tickle) is applied to the endcap of the ion trap to activate the target ion to induce collisional dissociation. With the development of fast personal computers, the speed for calculation of the time domain SWIFT waveform with a fast Fourier transform algorithm can be completed in less than $50 \mathrm{~ms}$. Thus it is possible to apply this technology to high efficiency separation techniques such as capillary HPLC, where the width of the peaks are typically less than $25 \mathrm{~s}$.

In this article, we report the development of an online capillary LC/MS/MS system by applying the SWIFT technology to the IT/reTOF system. Two computers are used here, one is used to perform data acquisition and analysis, the other to generate SWIFT and tickle voltages. The amplitude of the tickle voltage is optimized on-line with a feedback method through the communication between two computers. Thus two HPLC runs are used for obtaining the MS/MS information for selected analytes.

\section{EXPERIMENTAL SECTION}

\section{Liquid chromatography}

A Star 9012 solvent delivery system (Varian Associates, Inc., Walnut Creek, CA, USA) was used at a flow rate of $200 \mu \mathrm{L} / \mathrm{min}$. A prime/purge valve located before the injection valve splits the mobile phase with a ratio of 39:1 to accommodate capillary column HPLC. The reverse phase micro-capillary HPLC column $(0.25 \mathrm{~mm} \times 150 \mathrm{~mm})$ was packed in-house using the slurry packing method reported previously. ${ }^{31}$ Peptide mixtures were injected through a Valco sample injector (Model C6W, Valco Instruments Co., Houston, TX, USA). The separation was monitored with an in-line Star 9050 (Varian) variable wavelength UV detector at $214 \mathrm{~nm}$, which was digitized through an analog to digital converter board embedded in a 486-PC. Separations were performed with $0.1 \%$ TFA in water as solvent $\mathrm{A}$ and $0.1 \%$ TFA in acetonitrile as solvent $\mathrm{B}$. A linear gradient was employed for the LC/MS separation. Initially the separation starts with $100 \%$ A for 3 minutes to stack the sample on the front of the capillary column ( $250 \mu \mathrm{m}$ i.d., C-18 reversed phase), then B is ramped to $80 \%$ in $40 \mathrm{~min}$.

A low-pressure syringe pump (Model 55-2222, Harvard Apparatus, South Natick, MS, USA) delivered isocratic solvent through the sample injection valve to the electrospray interface in the direct injection experiments. The solvent used in the direct injection mode was methanol and the flow rate was typically set at $3 \mu \mathrm{L} / \mathrm{min}$. Single peptide samples were injected in this mode to optimize the mass spectrometer.

\section{Chemicals}

The peptides melittin, bradykinin, neurotensin, angiotensin III and leucine enkephalin-arginine were purchased from Sigma Chemical Company (St. Louis, MO, USA). HPLC grade acetonitrile and methanol was obtained from Aldrich Chemical Co. (Milwaukee, WI, USA). TFA was purchased from Pierce (Rockford, IL, USA). HPLC grade water was generated in-house with a Millipore Milli-Q water purification system (Bedford, MA, USA).

\section{Mass spectrometer}

The experimental configuration consists of a differentially pumped reflectron time-of-flight mass spectrometer (Model 850, R. M. Jordan Co., Grass Valley, USA) interfaced to a quadrupole ion trap (Model C-1251, R. M. Jordan Co.) described in previous work. ${ }^{33}$ The modifications to this mass spectrometer for on-line LC/MS analysis have also been previously reported..$^{20,21}$ The electrospray interface ${ }^{20}$ consists of a 26 gauge flat tipped stainless steel needle connected to a Valco zero dead volume tee. A fused silica tube (175 $\mu \mathrm{m}$ o.d., $75 \mu \mathrm{m}$ i.d.) was inserted through the needle to deliver sample and buffer solution to the spray head. The capillary protrudes from the needle by $\sim 1 \mathrm{~mm}$ to maintain a stable electrospray. The needle is floated to $\sim 3800 \mathrm{~V}$ relative to a heated $\left(\sim 120^{\circ} \mathrm{C}\right)$ stainless steel inlet tubing (1/16 in i.d., $0.5 \mathrm{~mm}$ o.d.). Droplets formed at the needle tip pass through the heated tubing and are desolvated in this process. The resulting ions are focused and sampled through an orifice $(325 \mu \mathrm{m})$ of a gold-plated skimmer. The ions are collimated by a set of Einzel lens towards a center aperture (3.1 mm dia.) on the entrance endcap of the ion trap and are trapped using an Ar buffer gas of $\sim 1$ mTorr in the trap. Two identical half-cylindrical electrodes $\left(\mathrm{E}_{\text {top }}, \mathrm{E}_{\text {bottom }}\right)$ are placed in the counter position to form the cylindrical center electrode of the Einzel lens. In the ion accumulation period $(400 \mathrm{~ms})$, 
both $E_{\text {top }}$ and $E_{\text {bottom }}$ are grounded and the ion beam can pass the Einzel lens. At the end of the ion accumulation period, a $+400 \mathrm{~V}, 100 \mathrm{~ms}$ long pulse is applied to $E_{\text {top }}$ to prevent ions from entering the ion trap. During this gating period the isolation and MS/MS activation processes may proceed without interference. After the $100 \mathrm{~ms}$ gating period, a negative extraction DC pulse $(-400 \mathrm{~V}, \sim 2 \mu \mathrm{s})$ is applied to the entrance endcap to eject ions into the time of flight (TOF) mass spectrometer.

\section{Data acquisition}

The data acquisition software has been developed in house using DOS-based Borland Pascal for speed and control. Mass spectra are digitized by a transient digitizer (Model PI9845, Precision Instruments Inc., Knoxville, TN, USA). The data system offset is set such that only meaningful ion intensities above a threshold result in non-zero data. Background noise $(90 \%$ of the data values) register as zero. The data is compressed in the computer into a point-pair representation of data point number and intensity. This permits an approximate reduction in data set size of 10:1 and allows each mass spectrum to be saved to a caching hard disk controller after it is acquired and real time graphics to be displayed. A typical total eluted chromatography data set is reduced from several tens of megabytes to several megabytes. Such entire data sets can be retained in computer memory for graphical analysis and 2D false color representation of mass spectrum vs. elution time. Using these techniques, we have demonstrated non-SWIFT spectral acquisition rates of $20 \mathrm{~Hz} .{ }^{34}$ The mass spectral acquisiton rate used in this study was 2 spectra/s, mainly limited by adequate signal to noise ratio. The maximum rate is $4 \mathrm{spectra} / \mathrm{s}$ for ions with high ionization efficiency.

\section{Communication between the two computers}

Identical digital input and output boards (PIO-24, Keithley Metrabyte, Taunton, MA, USA) are installed in each computer, respectively. The two boards are connected through a standard 36-pin ribbon cable. The data acquisition computer is operated as the master and the SWIFT generation computer as slave. The data acquisiton computer acquires mass spectra, performs analysis, makes decisions and then generates commands to the I/O ports of the CIO/DIO24 board. The SWIFT generation program reads these values and responds accordingly.

\section{Hardware for SWIFT waveform generation}

The SWIFT waveform is calculated in real time on a second computer (Pentium 200, Gateway 2000, North Sioux City, SD, USA) and loaded to a PC-compatible arbitrary waveform generation board (Model PCIPAWFG, Keithley Metrabyte). The arbitrary waveform generator (AWFG) board can generate waveforms at a maximum update rate of $5 \mathrm{MHz}$ with 12 bit amplitude resolution. Its maximum voltage range is $-10 \mathrm{~V}$ to $10 \mathrm{~V}$. The maximum amount of data which can be loaded in its RAM is $64 \mathrm{~kb}$ which makes it possible to generate $32 \mathrm{k}$ points in one waveform ( 2 bytes/point). As a compromise between the requirement for fast data acquisition and the time necessary for waveform calculation and loading, we have chosen a waveform size of 8192 points and an update rate of $2.5 \mathrm{MHz}$ as our operating condition.

\section{SWIFT and tickle waveform design}

The SWIFT waveform, which is used for isolating an ion of known $m / z$, is constructed by carrying out the following steps: (A) calculate the secular frequency of the ion movement inside the ion trap. When the value of $q_{\mathrm{z}}$ is less than $0.4, \beta_{\mathrm{z}} \approx\left(\mathrm{a}_{\mathrm{z}}+\left(q_{\mathrm{z}}^{2} / 2\right)\right)^{1 / 2}$ and $\omega_{\mathrm{z}}=$ $0.5 *\left(\Omega^{*} \beta_{\mathrm{z}}\right)$, where $\Omega$ stands for the frequency of the RF voltage applied to the ion trap ring electrode, $\omega_{\mathrm{z}}$ stands for the secular frequency of the ion, $a_{\mathrm{z}}$ is a factor determined by the DC voltage applied to the ring electrode, and $q_{\mathrm{z}}$ is a factor determined by the amplitude of the RF voltage. (B) Build a magnitude spectrum from $10 \mathrm{kHz}$ to $250 \mathrm{kHz}$ as shown in Fig. 1(a). This corresponds to a $m / z$ range from $113 \mathrm{Da}$ to $2817 \mathrm{Da}$ (the low mass cut-off is $114 \mathrm{Da}$ under the current setup), the frequency of the center of the notch is the secular frequency calculated in (A), the width of the notch is set at $2.5 \mathrm{kHz}$. (C) To transform the magnitude spectrum to the time domain and reduce the maximum amplitude of the SWIFT waveform, phase modulation is necessary. The phase modulation method employed is termed quadratic phase modulation. ${ }^{26,27,29}$ The time-domain SWIFT waveform obtained with this method concentrates most of the SWIFT signal to the center of the time domain interval (Fig. 1(b)).

Three sine waveforms of different frequencies are added together and normalized to construct the tickle waveform. The frequencies $(\mathrm{Hz})$ of these three waveforms are: $\left(\omega_{z}-500\right), \omega_{z},\left(\omega_{z}+500\right)$. The initial phases of these three sine waveforms are modulated using the same method as described above. 1024 points are used to represent one time interval of the synthesized tickle waveform. Under the current waveform output update rate of the AWFG board $(2.5 \mathrm{MHz})$, it is necessary to repeatedly generate that same interval in order to extend the CID activation time to $\sim 33 \mathrm{~ms}$.

Due to the limited time available to calculate the SWIFT waveform data and load this large amount of data from the memory of the computer to the RAM of the AWFG board, we generate a SWIFT waveform containing much less than the maximum $32 \mathrm{k}$ possible points that the AWFG board can hold. The short SWIFT waveform is repeated 10 times after the gating starts to extend the isolation period to around $33 \mathrm{~ms}$. After isolation, the output voltage of the AWFG board is set to $0 \mathrm{~V}$ for a cooling period of $22 \mathrm{~ms}$ to let the isolated ions relax. The tickle waveform is loaded into the AWFG memory in this interval and applied to the entrance endcap immediately following the cooling period. The total isolation-cooling-tickling process requires about $88 \mathrm{~ms}$. The DC extraction pulse is applied to the exit endcap $12 \mathrm{~ms}$ later. For this existing method, the SWIFT and tickle waveforms are each loaded into the AWFG board for each mass spectrum obtained. This time consuming process is one limitation to the maximum sampling rate.

The commands from the master computer set the slave computer to one of three possible states: (1) 
generating SWIFT and tickle waveforms; (2) generating only SWIFT; (3) waiting at an output of $0 \mathrm{~V}$. For each mass spectrum, the master computer controls whether the tickle voltage needs to be increased, decreased or has been optimized and is held constant.

\section{Protocols for performing on-line LC/MS/MS}

In order to perform on-line analysis, two sample injections are required. The first is used to identify and select the parent ion to monitor in the subsequent injection in which MS/MS fragmentation occurs for identification of that parent ion. From the LC/MS data of the first injection, an integrated total ion chromatogram (TIC) is obtained along with a 2-D false color image of mass spectra vs. elution time. The image pixel color is varied according to the intensity of the ion peak at a given $\mathrm{m} / \mathrm{z}$ ratio. From this LC/MS TIC and 2-D image we select, for each eluted peak in the TIC, which ion to monitor following the second injection. A small $\mathrm{m} / \mathrm{z}$ range spanning this analyte ion $\mathrm{m} / \mathrm{z}$ is chosen. The integrated ion intensity over this selected ion range (selected ion chromatography, SIC) will initially be monitored to determine when that particular peak has begun to elute. Then that intensity will be used to determine the efficiency of fragmentation for a particular applied tickle voltage.

Two sets of data are entered into the two computers.
The first is called the peak-detection data set and is entered into the data acquisition computer. For each ion to be monitored in the second injection for on-line LC/MS/MS analysis we enter four parameters: (1) time of the SIC integration starting point; (2) time of the SIC integration ending point; (3) threshold for peakdetection (the SIC amplitude); (4) the TIC peak width of the ion. The second set of data is called the SWIFT generation data. For each analyte ion we enter values for five parameters: (1) the secular frequency of the ion; (2) the width of the notch that is used when constructing the magnitude spectrum; (3) the high limit of the tickle voltage maximum amplitude (HMV); (4) the low limit of the tickle voltage maximum amplitude (LMV); (5) the initial maximum amplitude (AMV).

In a second HPLC analysis the master computer tells the slave computer to begin generating SWIFT waveforms without a tickle voltage several minutes before the HPLC peak of ion 1 elutes (this time is approximated from the first run). The master computer will analyze every spectrum and perform SIC integration. If the integration SIC value of one mass spectra (spectrum 1) becomes larger than the preset threshold for peak detection, then the program recognizes that ion 1 has eluted. Subsequently it sets the slave computer to generate both SWIFT and tickle waveforms on the next spectra. After it collects the next spectrum (spectrum 2)
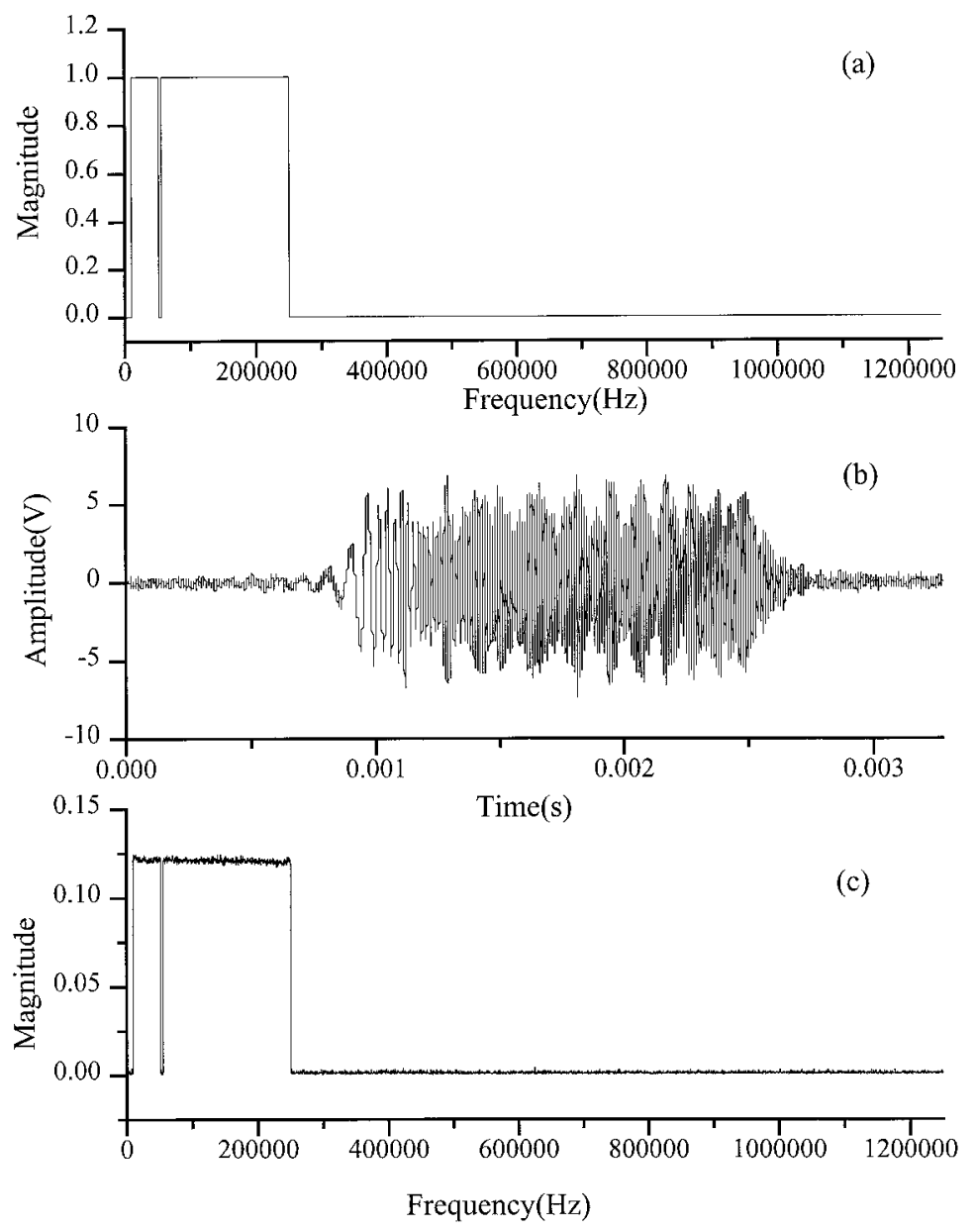

Figure 1. (a) The magnitude spectrum of the SWIFT waveform, (b) the SWIFT waveform output from the AWFG board and sampled using a digital oscilloscope, (c) the magnitude spectrum by inverse Fourier transform of the SWIFT waveform in (b). 
and does SIC integration over the same interval, this second integration value is divided by the integration value from spectra 1 . If the resulting value $(\delta)$ falls in a preset range (usually $0.05-0.15$ ), the master computer recognizes that the fragmentation efficiency is optimized and sets the slave computer to then constantly generate the tickle voltage at the current value. If $\delta$ is above the range, the master computer informs the slave to generate another SWIFT waveform without tickle when it acquires the next mass spectrum (spectrum 3), and generate a SWIFT waveform plus tickle with increased maximum amplitude when it acquires the fourth mass spectrum (spectrum 4). It is necessary to generate another spectrum without tickle voltage (spectrum 3) because the compound is eluting and the SIC will now be greater than in spectra 1 . The slave computer increases the maximum amplitude of the tickle waveform using the following assignments when it acquires spectra 4 :

(a) $\mathrm{LMV}:=\mathrm{AMV}$

(b) $\mathrm{AMV}:=(\mathrm{LMV}+\mathrm{HMV}) / 2$

If $\delta$ is lower than the preset range, it means that the tickle voltage is too high, the slave computer will change the amplitude of the tickle waveform using the following assignments:
(a) HMV: = AMV
(b) $\mathrm{AMV}:=(\mathrm{LMV}+\mathrm{HMV}) / 2$

The SIC integration value obtained in spectra 3 and 4 are used to calculate a new value of $\delta$ and the same comparision scheme continues until $\delta$ falls in the preset range.

The algorithm presented above is actually a feedback, self-optimization algorithm. Initially LMV and $\mathrm{HMV}$ are set to 1 and $4 \mathrm{~V}_{\mathrm{P}-\mathrm{P}}$ respectively. Since each comparison will reduce the output range (HMV-LMV) by half, at most 11 comparisons are necessary to optimize the tickle voltage and the output range will be reduced to $\sim 0.03 \mathrm{~V}$ since each comparison will reduce the range by half. Further comparisons are not necessary since the resolution of the board is only 12 bits $(0.0244 \mathrm{~V}$ for a $10 \mathrm{~V}$ range $)$.

\section{RESULTS AND DISCUSSION}

Figure 1 shows the SWIFT waveform generation process. A flat-top magnitude spectrum (Fig. 1(a)) is generated between $10 \mathrm{kHz}$ and $250 \mathrm{kHz}$ with a notch whose center frequency equals the secular frequency. Another set of data generated are the initial phases corresponding to each frequency component in the magnitude spectrum using the quadratic phase modulation method. The magnitude spectrum data (magnitude vs. frequency) and the initial phase data (phase vs. frequency) are combined together to obtain the timedomain SWIFT waveform. The SWIFT waveform generated from the AWFG board is displayed in Fig. 1(b). The starting and ending edges of the SWIFT waveform are minimized because of the non-linear phase modulation. The AWFG waveform is converted by Fourier transform to the frequency domain as shown in Fig. 1(c). In Fig. 1(c) the magnitude spectrum is not

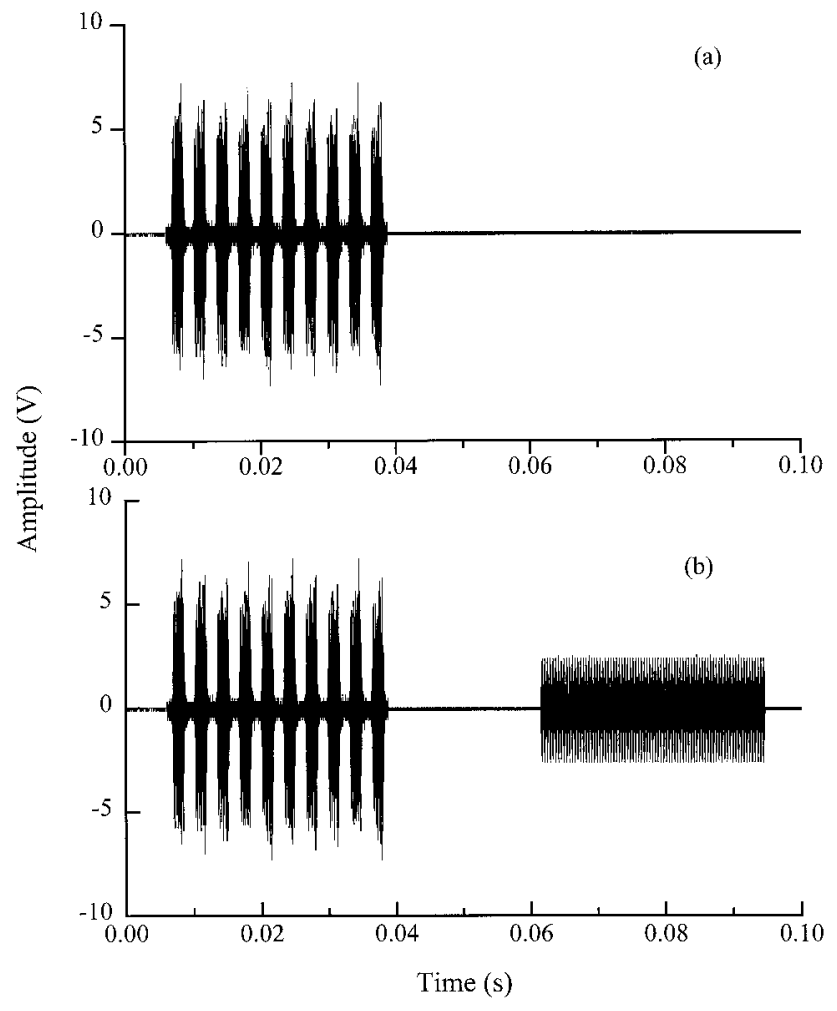

Figure 2. The typical sequence of waveforms used for (a) performing isolation, (b) performing isolation and resonant CID activation.

as flat as in Fig. 1(a) because of the limited resolution (12 bit) of the AWFG board output. The actual magnitudes of different frequency components fall within a narrow band across the top of the magnitude spectrum shown in Fig. 1(c). This shows that there are small variations in excitation energy across the activation

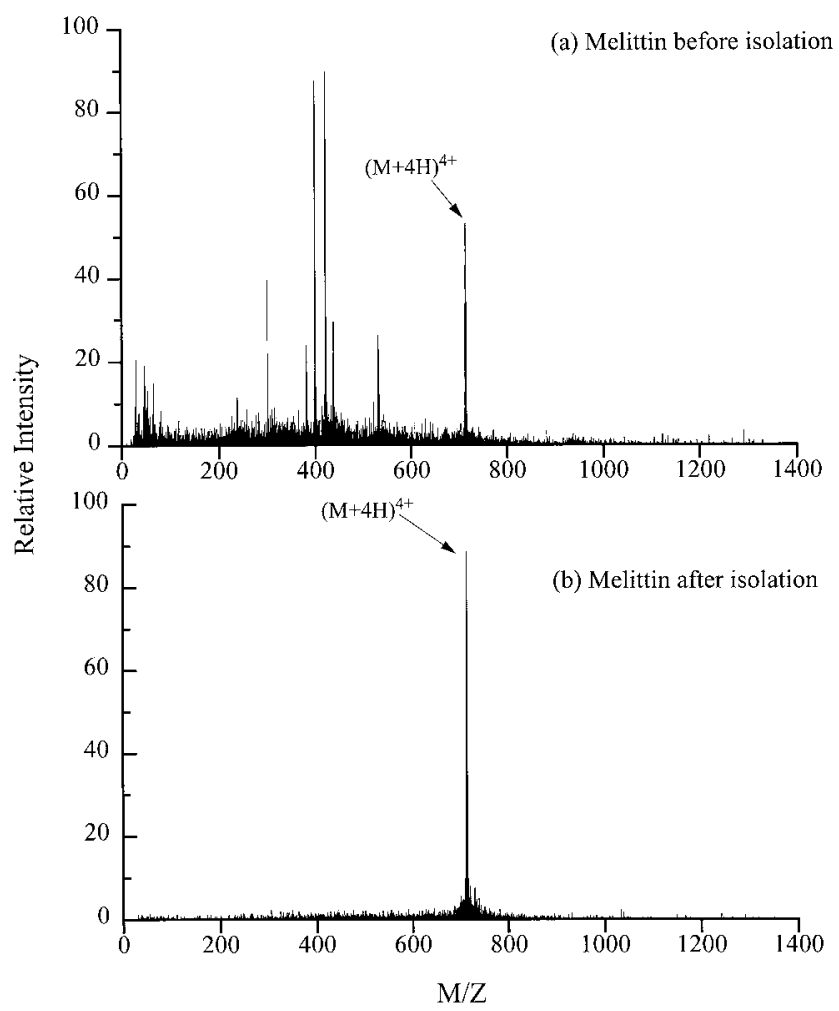

Figure 3. The mass spectrum of mellitin (a) before isolation, (b) after isolation using SWIFT. 


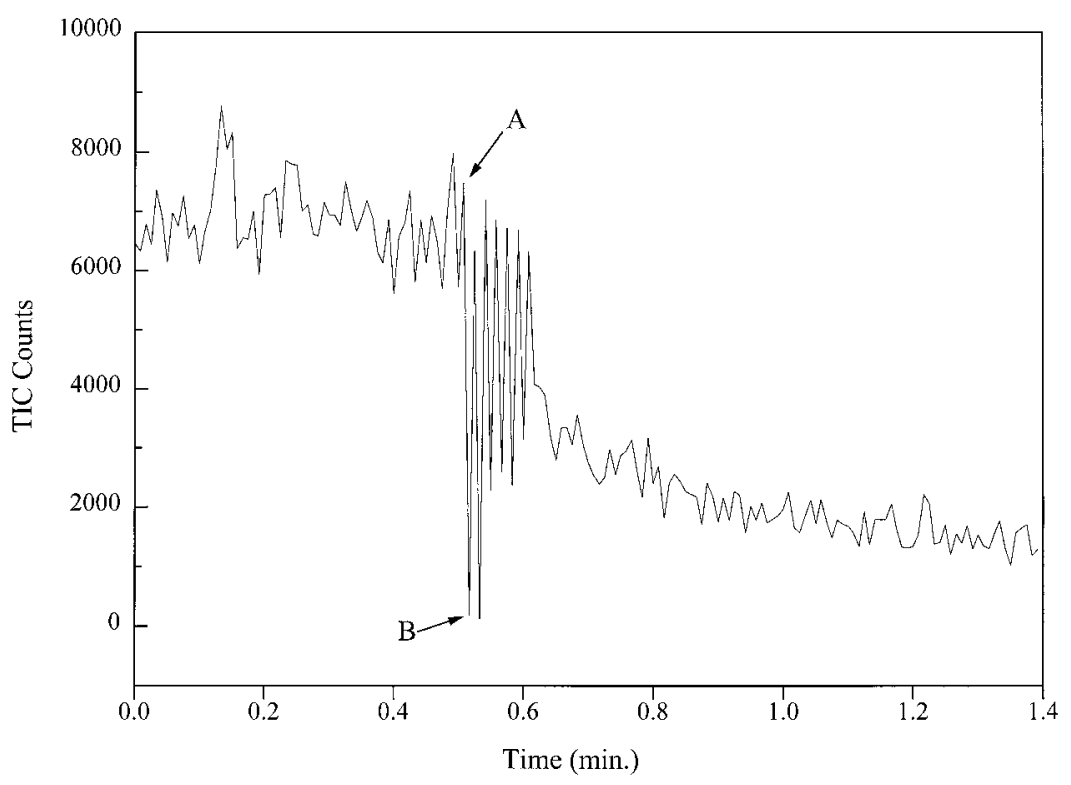

Figure 4. The TIC of the mass spectra collected in the self-optimization process.

frequency range. By extending the isolation time, this effect becomes negligible because ions with less excitation can eventually obtain sufficient energy to be ejected.

The duration for a single SWIFT waveform is only about 3.2. It has been reported in previous work ${ }^{23}$ that an isolation time of $25 \mathrm{~ms}$ is required to eject all of the unwanted ions. The typical isolation and MS/MS timing sequence used in this work is displayed in Fig. 2(a) and (b). Under current experimental conditions, we determined that consecutively generating ten SWIFT waveforms is sufficient to eject all the unwanted ions with discrimination against ions of close $m / z$ values. Because the secular frequency calculated using the equation described previously is within $\pm 2 \%$ of the experimental value, ${ }^{23}$ we enlarged the isolation window

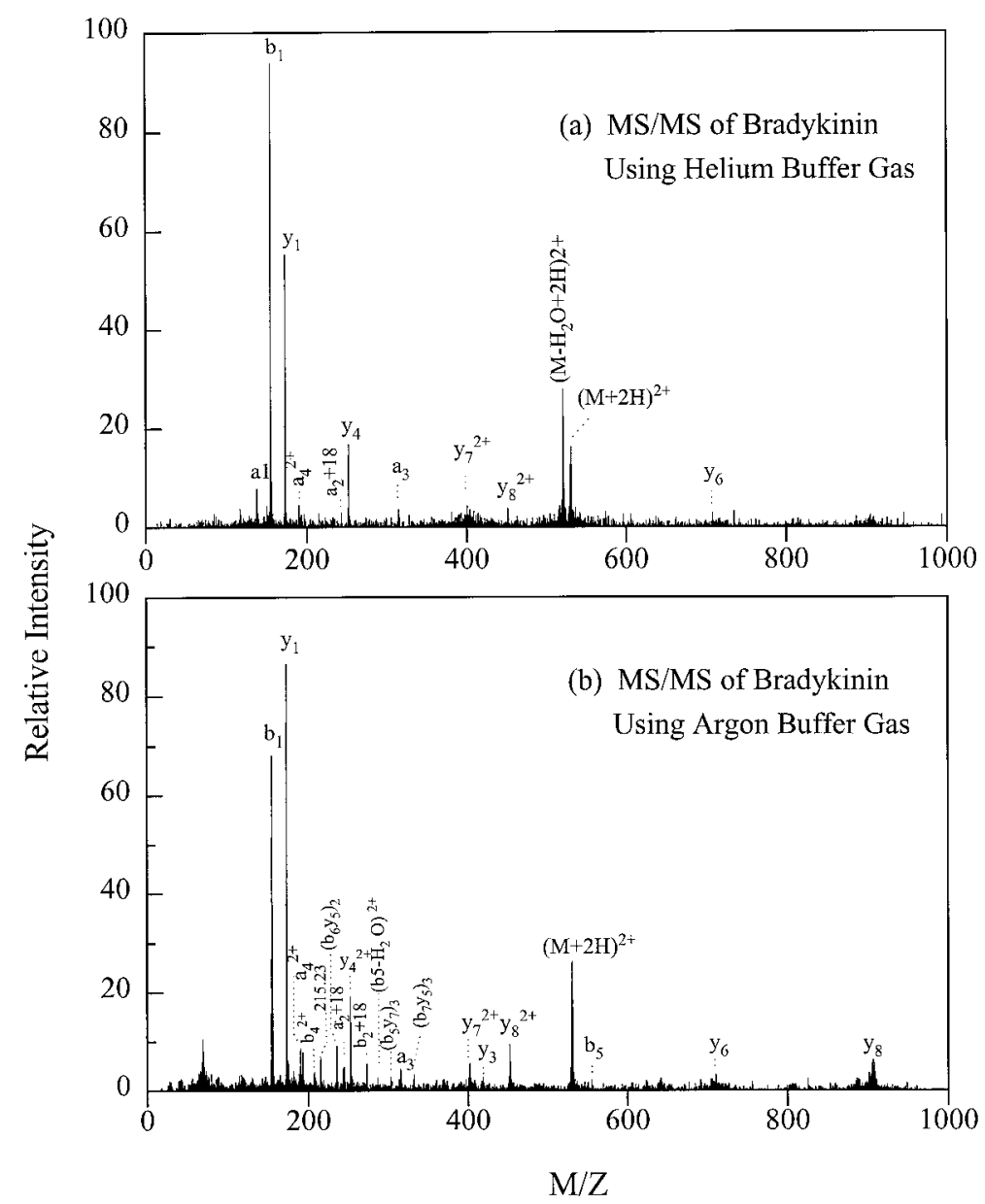

Figure 5. The MS/MS of bradykinin (a) helium buffer gas, (b) argon buffer gas. 


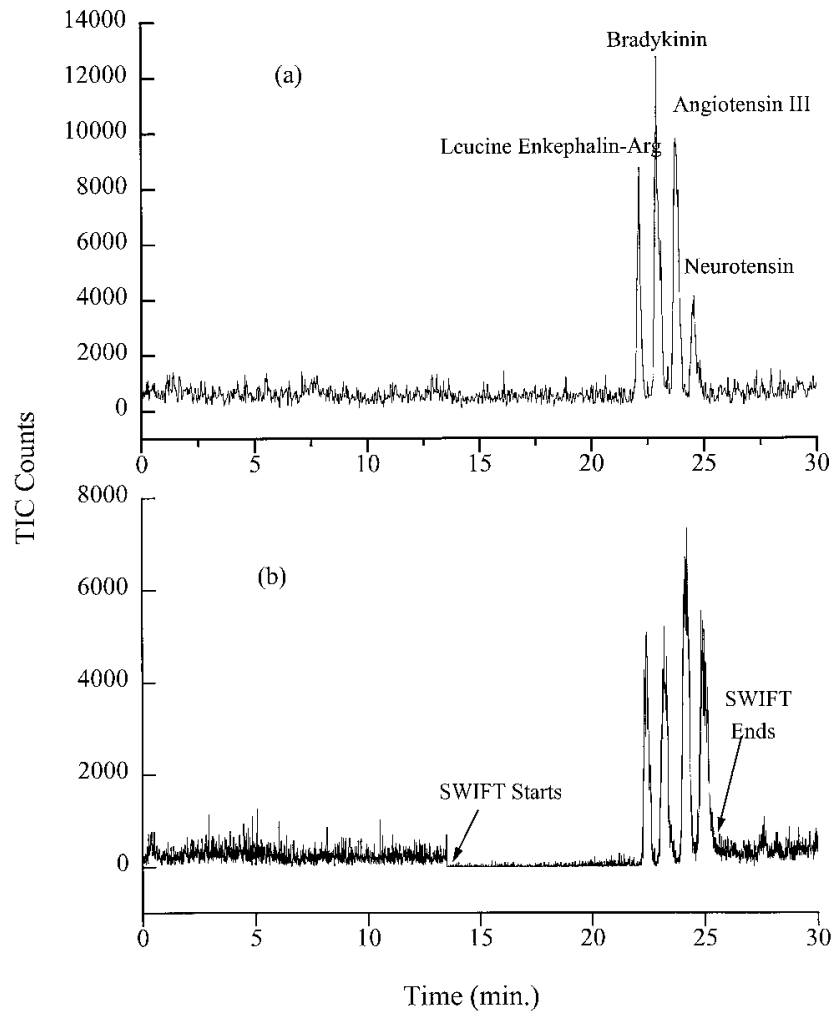

Figure 6. (a) TIC of the LC/MS for the first injection, (b) TIC of the $\mathrm{LC} / \mathrm{MS} / \mathrm{MS}$ for the second injection.

(notch width $\sim 2.5 \mathrm{kHz}$ ) so that small variations in the secular frequencies will not greatly reduce the isolation efficiency. One observation in our experiments is that the width of the notch is related to the maximum amplitude of the isolation SWIFT waveform. A narrower width requires a lower maximum amplitude. In the direct injection mode, the notch can be narrowed to about $0.8 \mathrm{kHz}$ by manually fine tuning the center frequency of the notch so that a mass window of $\pm 6 \mathrm{u}$ can be detected. However, the amplitude was not sufficiently large to eject all the unwanted ions in the $33 \mathrm{~ms}$ isolation time interval. When the notch width is sufficiently large $(\geq 2.5 \mathrm{kHz})$, the amplitude of the SWIFT waveform does not significantly influence the signal intensity of the target ion. To optimize the IT/ reTOF system for on-line analysis, we have experimentally chosen a notch width of $2.5 \mathrm{kHz}$ and a maximum amplitude of $7.5 \mathrm{~V}$ for all the experiments performed.
Figure 3(a) shows the mass spectrum of ESI generated melittin ions (MW 2848) obtained in the direct injection mode without ion isolation. There are many low mass ions generated in the skimmer CID process and the use of Ar buffer gas also contributes to the extensive fragmentation. In Fig. 3(b) is shown a mass spectrum acquired after applying the isolation sequence shown in Fig. 2 to the entrance end cap. The signal to noise ratio of the target ion has been improved by a factor $\sim 5$ due to ejection of unwanted ions.

An important factor which must be optimized to perform on-line MS/MS experiments is the amplitude of the tickle waveform. In initial experiments we found that fragmentation efficiency is very sensitive to its maximum amplitude. For larger peptides, such as neurotensin, the range of optimal activation voltage is within $0.02 \mathrm{~V}$. For some smaller peptides such as leucine-enkephalin this range is about $0.1 \mathrm{~V}$. To perform LC/MS/MS, the tickle voltage must be optimized on-line. Currently we are using the feed-back comparison model described above to optimize the activation voltage. In Fig. 4 is shown the TIC for a typical optimization process. One half minute after SWIFT isolation begins, the master program starts (point A) testing to see if the target ion's mass peak exists or not. If the SIC integration value is above the preset threshold (which is the case here) the self-optimizing process starts (point A). The master computer then tells the slave computer to generate the tickle waveform at the preset initial activating voltage. The SIC value of the next spectrum (point $\mathrm{B}$, tickle on) is then divided by the value at point A (tickle off) to decide whether to increase or decrease the tickle voltage. This comparison continues until the ratio falls in a preset range, $0.05-0.15$, which indicates that approximately $85-95 \%$ of the target ions have been fragmented (point $\mathrm{C}$ ). The averaged MS/MS spectrum is shown in Fig. 5(a) and (b), using different buffer gases.

The effects of helium buffer gas have also been examined as a factor for introducing fragmentation under the self-optimization scheme as compared to argon. The actual pressures of $\mathrm{He}$ and $\mathrm{Ar}$ inside the ion trap are estimated to be in the range of $1 \times 10^{-4}$ to $2 \times 10^{-3}$ Torr. The flow rates of the two buffer gases were optimized independently to obtain the best MS/ MS spectrum in each case. Figure 5 shows two MS/MS spectra of bradykinin using Ar and He buffer gases, separately. It is observed that the Ar buffer gas

Table 1. List of peptides and their initial parameters for peak detection, tickle self-optimization, and the optimal tickle voltage obtained on-line

$\begin{array}{lc} & \text { MW }^{a} \\ \text { Leu-EnkArg } & 711.3704 \\ \text { Bradykinin } & 1059.561 \\ \text { Angiotensin III } & 930.5075 \\ \text { Neurotensin } & 1671.91\end{array}$

$\begin{array}{cc}\mathrm{M}_{\mathrm{CID}}{ }^{b} & \mathrm{MW}^{\mathrm{c}} \\ 1+ & 711.8 \\ 2+ & 530.1 \\ 2+ & 465.6 \\ 3+ & 557.6\end{array}$

$\begin{array}{rr}\mathrm{t}_{1}{ }^{d} & \mathrm{t}_{2}{ }^{e} \\ 105.0 & 107.0 \\ 90.5 & 92.5 \\ 85.5 & 87.5 \\ 114.0 & 116.0\end{array}$

$\begin{array}{cl}\mathrm{h}^{f} & \mathrm{f}_{\mathrm{z}}^{g} \\ 500 & 39.56 \\ 500 & 53.1 \\ 500 & 60.49 \\ 300 & 50.51\end{array}$

HMV
4.00
4.00
4.00
4.00

Monoisotopic molecular weight.

' SIC integration ending time ( $\mu$ s).

The threshold for detecting the peak (counts).

${ }^{8}$ The secular frequency of the precursor ion $(\mathrm{kHz})$.

"The optimal tickle voltage $\left(\mathrm{V}_{\mathrm{O}-\mathrm{P}}\right)$ obtained in the second injection. 
produces more intense fragmentation in both the low and high mass regions. The Ar buffer gas results in a higher energy deposition and the sample ions can overcome the fragmentation energy threshold. In general, Ar buffer gas has been used for on-line analysis in this work.

HPLC/MS/MS is demonstrated for a mixture of four peptides in Fig. 6. In the first step we performed HPLC/ MS separation and detection. The TIC is shown in Fig. 6(a). For each peptide, we calculate the $m / z$ value of a singly or multiply charged mass peak whose intensity is the strongest compared to other molecular ions of different charge states. The useful information retrieved from LC/MS spectra and the initial condition for the MS/MS experiment are shown in Table 1. These initial conditions were entered into the computer that performs data acquisition and the computer that generates the SWIFT waveform. After injecting $4 \mu \mathrm{L}$ of the peptide mixture $\left(\sim 1 \times 10^{-5} \mathrm{M}\right)$, both programs were initiated. The TIC of the LC/MS/MS experiment is shown in Fig. 6(b) and the resulting MS/MS spectra for each peptide are shown in Fig. 7(a)-(d). Because of the time required to optimize the tickle voltage $(\sim 10 \mathrm{~s})$, the $\mathrm{S} / \mathrm{N}$ ratio of the $\mathrm{LC} / \mathrm{MS} / \mathrm{MS}$ is lower compared to

(a)

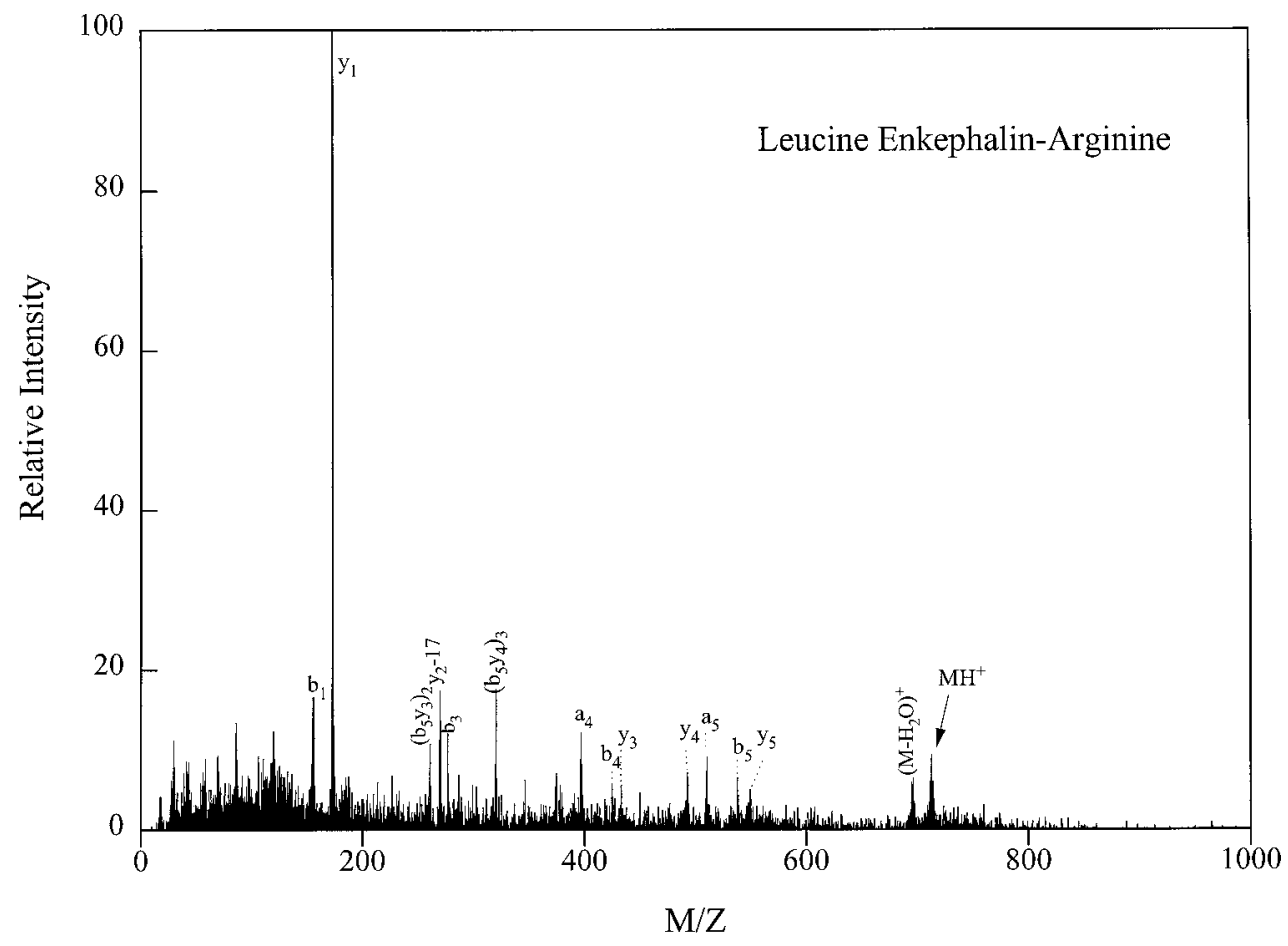

(b)

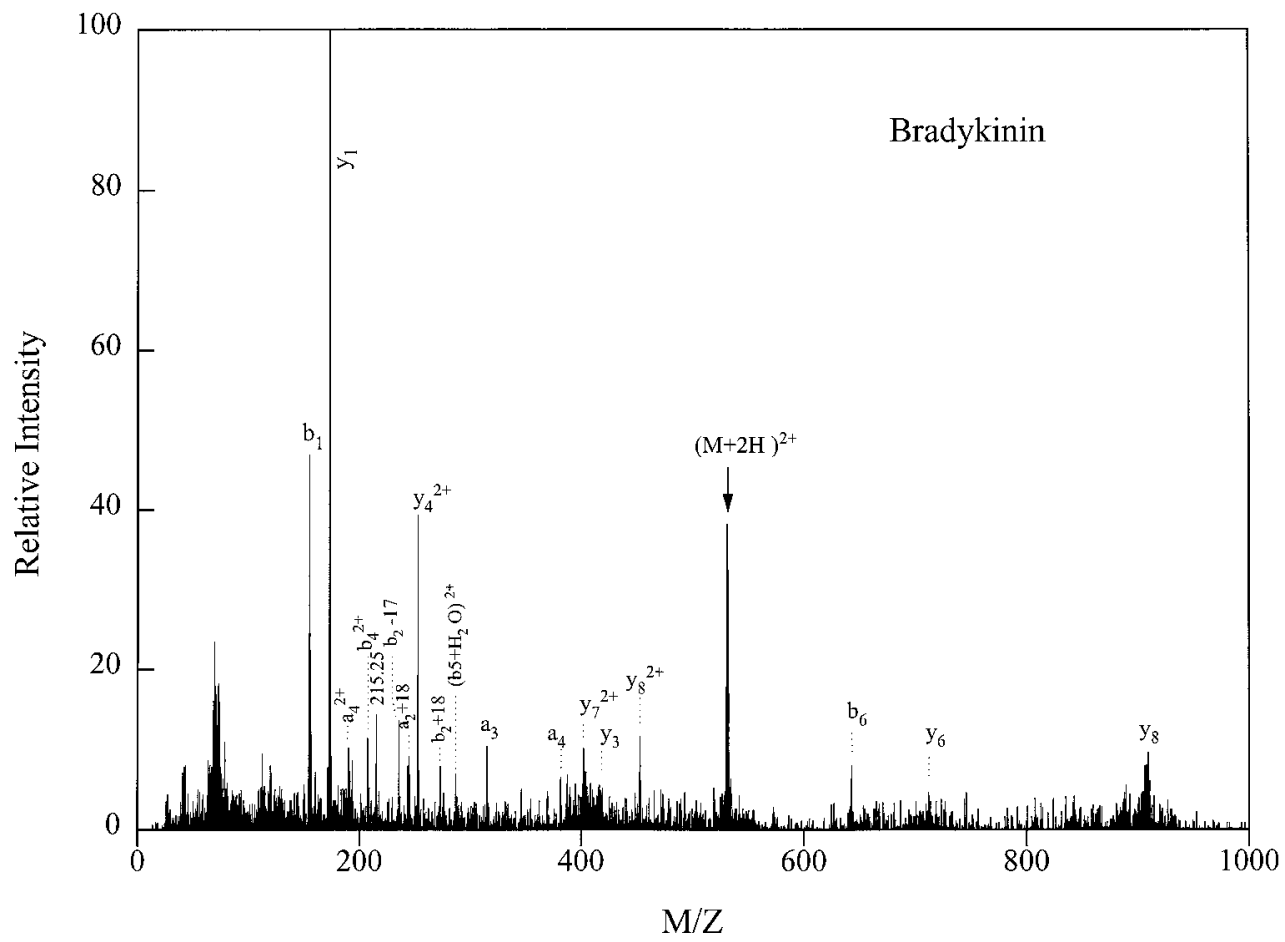

Figure 7. Continued over. 
the direct injection MS/MS spectra due to limited (5-15) single shot MS/MS spectra available for averaging. The TFA added to the HPLC mobile phase reduces the signal intensity and this effect reduces the $\mathrm{S} / \mathrm{N}$ of the MS/MS spectra due to limited (5-15) single shot MS/MS spectra available for averaging. The TFA added to the HPLC mobile phase reduces the signal intensity and this effect reduces the $\mathrm{S} / \mathrm{N}$ of the MS/MS spectra accordingly. A relatively complete sequence of the $b$ and $\mathrm{y}$ ions have been observed for neurotensin, bradykinin and leucine enkephalin-arginine. In the case of angiotensin III, the lack of fragmentation in the high mass region and the existence of strong low mass peaks possibly indicates that the pressure of buffer gas is too high.

The impact of HPLC peak shape on tickle optimization has been examined. The optimization process occurs on the rising edge of the peak while the MS/MS spectra are mostly collected at the center and falling side of each peak. The tickle voltage optimization

(c)

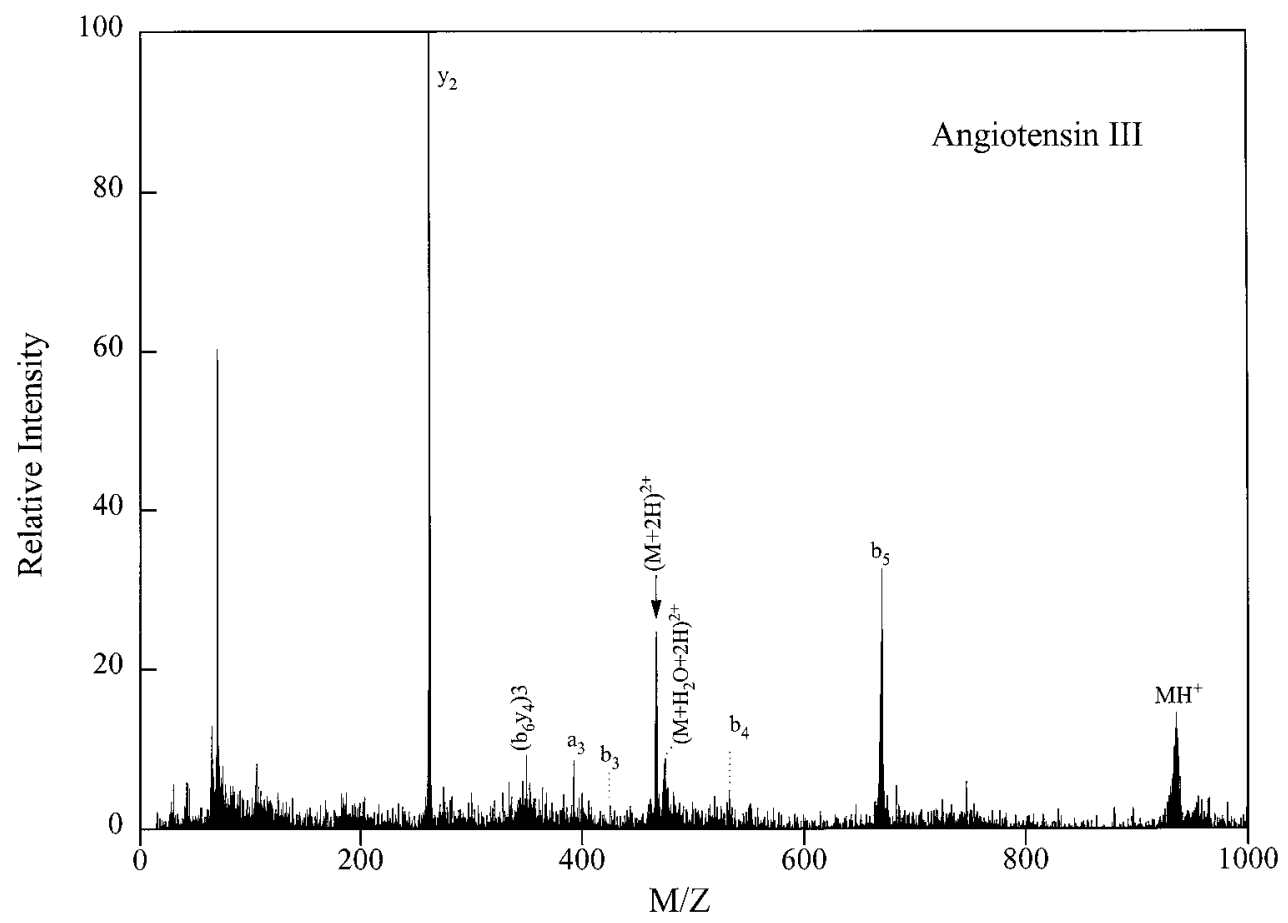

(d)

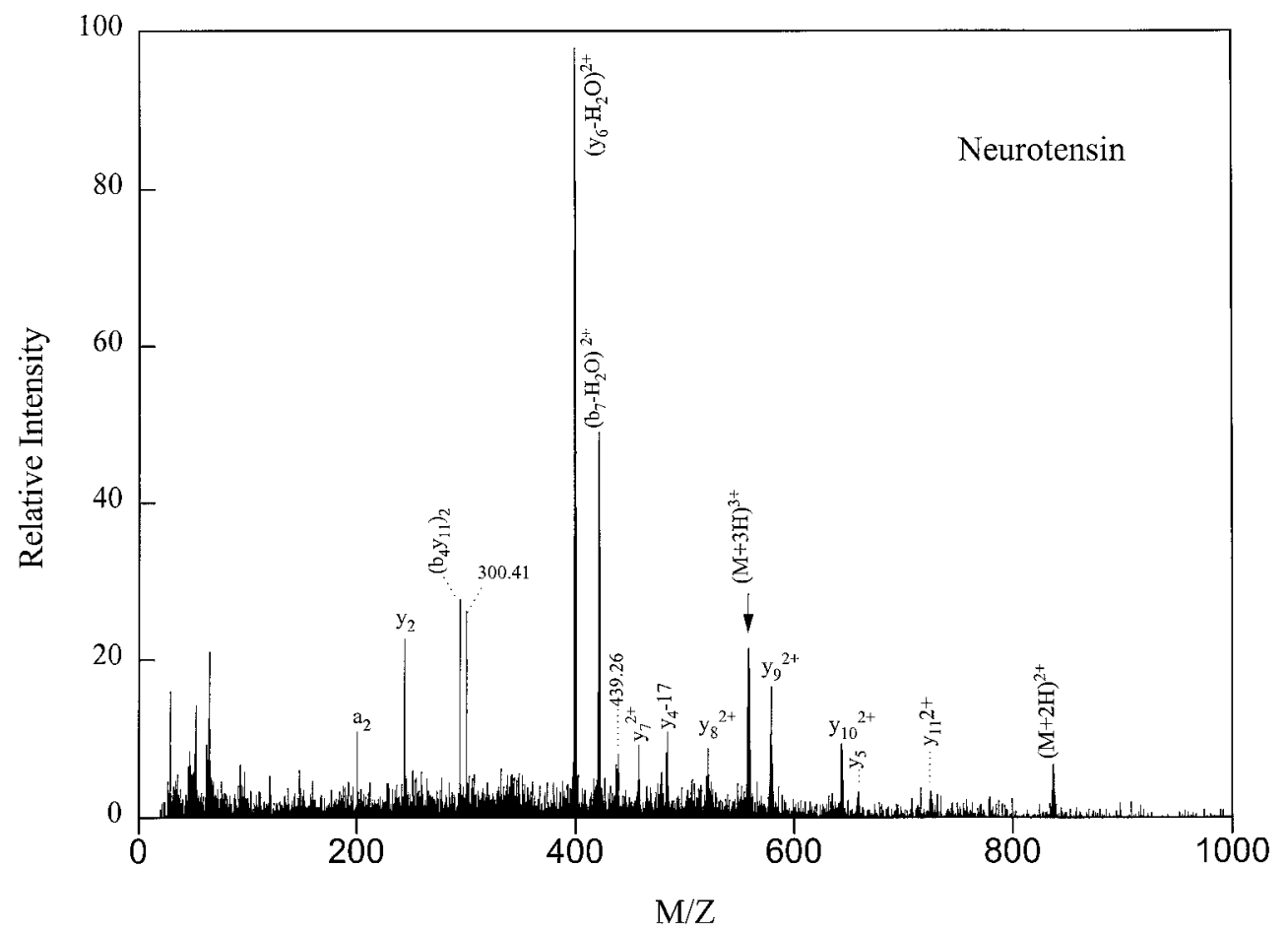

Figure 7. (a) MS/MS spectrum of leucine enkephalin-arginine, the arrow indicates the precursor ion, (b) MS/MS spectrum of bradykinin, the arrow indicates the precursor ion, (c) MS/MS spectrum of angiotensin III, the arrow indicates the precursor ion, (d) MS/MS spectrum of neurotensin, the arrow indicates the precursor ion. 
method presented here has two problems. First, two spectra are required for comparison. This increases the time required for optimization and reduces the number of tickle-optimized MS/MS spectra that can ultimately be averaged. Second, shot-to-shot variations and concentration changes from the rising edge of the peak result in incorrect comparisons. The rising edge can be accounted for by increasing both the lower and higher limit $(0.05$ to 0.15$)$ of the optimal range to result in a more optimal final tickle voltage.

Optimum tickle voltage occurs when most $(\sim 90 \%)$ of the selected parent ion is fragmented into detectable daughter ions useful for compound identification. By examining each spectrum individually and making an internal comparison over these two $m / z$ ranges, the optimal tickle voltage can be more properly and quickly identified. Initial work using this second approach appears to reduce the optimization time by one-half. ${ }^{35}$ Shot-to-shot variations of the mass spectra will have less effect on this ratio. Other algorithms which involve varying the tickle voltage during optimization are being explored. Additionally, other hardware and methods for waveform and tickle voltage generation are being evaluated. These improvements will decrease the number of spectra required for tickle voltage optimization and increase the spectral acquisition rate to the point that this method can be successfully applied to the significantly narrower peaks observed in capillary electrophoresis.

\section{CONCLUSION}

The HPLC/MS/MS capabilities of a quadrupole ion trap storage/reflectron time-of-flight mass spectrometer have been demonstrated. The isolation of target ions was achieved by calculating and generating SWIFT waveforms in real time. The optimal activation voltage was obtained on-line by employing a feed-back selfoptimization protocol. The LC/MS/MS was achieved in two HPLC runs, where the LC/MS data collected in the first run can be used to set the initial conditions for the tandem mass experiment performed in the second run. This system has been tested with a mixture composed of 4 peptides. The experiments have shown that SWIFT technology can be applied for on-line capillary HPLC/ MS/MS analysis of peptide mixtures in the ion trap storage/reflectron time-of-flight hybrid mass spectrometer. The high efficiency associated with ion dissociation and fragment-ion collection with the trap, ${ }^{24,29}$ the ability to detect ions in a non-scanning mode and the real time SWIFT waveform generation provides an alternative method to acquire sequence specific information of polypeptides as compared to scanning mass detection devices such as quadrupoles or the ion trap mass spectrometer.

\section{Acknowledgement}

We gratefully acknowledge support of this work by the National Institute of Health under Grant Number R01GM49500 and the National Science Foundation under Grant Number BIR-9513878.

\section{REFERENCES}

1. J. B. Fenn, M. Mann, C. K. Meng, S. F. Wong and C. M. Whitehouse, Science 64, 246 (1989).

2. J. B. Fenn, M. Mann, C. K. Meng, S. F. Wong and C. M. Whitehouse, Mass Spectrom. Rev. 9, 37 (1990).

3. R. D. Smith, J. A. Olivares, N. T. Nguyen and H. R. Udseth, Anal. Chem. 60, 436 (1988).

4. J. A. Loo, H. R. Udseth and R. D. Smith, Anal. Biochem. 179, 404 (1989).

5. E. C. Huang, T. Wachs, J. J. Conboy and J. D. Henion, Anal. Chem. 62, 713A (1990).

6. S. K. Chowdhury, V. Katta and B. T. Chait, Rapid Commun. Mass Spectrom. 4, 81 (1990).

7. J. A. Loo, C. G. Edmonds and R. D. Smith, Anal. Chim. Acta 241, 167 (1990).

8. C. K. Meng, C. N. McEwen and B. S. Larsen, Rapid Commun. Mass Spectrom. 4, 151 (1990).

9. V. Katta, S. K. Chowdhury and B. T. Chait, Anal. Chem. 63, 174 (1991).

10. B. M. Chien and D. M. Lubman, Anal. Chem. 66, 1630 (1994).

11. C. J. Barinaga, C. G. Edmonds, H. R., Udseth and R. D. Smith, Rapid Commun. Mass Spectrom. 3, 160 (1989).

12. X. J. Tang, P. Thibault and R. K. Boyd, Anal. Chem. 65, 2824 (1993).

13. K. M. Downward and K. Biemann, J. Am. Soc. Mass Spectrom. 5, 966 (1994).

14. D. F. Hunt, N. Zhu and J. Shabanowitz, Rapid Commun. Mass Spectrom. 3, 122 (1989).

15. M. W. Senko, J. P. Speir and F. W. McLafferty, Anal. Chem. 66, 2801 (1994).

16. F. Vedel, M. Vedel and R. E. March, Int. J. Mass Spectrom. Ion Processes 108, R11 (1991).

17. S. A. Mcluckey, D. E. Georinger and G. L. Glish, Anal. Chem. 64, 1455 (1992).

18. S. A. Mcluckey, G. L. Van Berkel, G. L. Glish, E. C. Huang and J. D. Henion, Anal. Chem. 63, 375 (1991).

19. S. M. Michael, B. M. Chien and D. M. Lubman, Anal. Chem. 65 2614 (1993).

20. M. G. Qian and D. M. Lubman, Anal. Chem. 67, 2870 (1995).

21. M. G. Qian, K. Zheng, Y. Chen, C. L. Chang, S. M. Hanash and D. M. Lubman, Rapid Commun. Mass Spectrom. 10, 1079 (1996).

22. M. X. Li, L. Liu, J. T. Wu and D. M. Lubman, Anal. Chem. 69, 2451 (1997).

23. M. G. Qian and D. M. Lubman, Rapid Commun. Mass Spectrom. 10, 1991 (1996).

24. M. G. Qian and D. M. Lubman, Rapid Commun. Mass Spectrom. 9, 1275 (1995).

25. A. G. Marshall and T. L. Ricca, J. Am. Chem. Soc. 107, 7893 (1985).

26. L. Chen, T.-C. L. Wang and A. G. Marshall, Anal. Chem. 59, 449 (1987).

27. R. K. Julian and R. G. Cooks, Anal. Chem. 65, 1827 (1993).

28. M. H. Soni and R. G. Cooks, Anal. Chem. 66, 2488 (1994).

29. V. M. Doroshenko and R G. Cotter, Rapid Commun. Mass Spectrom. 10, 65 (1996).

30. D. E. Georinger, K. G. Asano, S. A. McLuckey, D. Hoekman and S. W. Stiller, Anal. Chem. 66, 313 (1994).

31. A. W. Garrett, M. E. Cisper, N. S. Nogar and P. H. Hemberger, Rapid Commun. Mass Spectrom. 8, 174 (1994).

32. A. V. Mordehai and J. D. Henion, Rapid Commun. Mass Spectrom. 7, 1131 (1993).

33. S. M. Michael, B. M. Chien and D. M. Lubman, Anal. Chem. 65, 2614 (1993).

34. M. G. Qian, J.-T. Wu, S. Parus and D. M. Lubman, Rapid Commun. Mass Spectrom. 10, 1209 (1996).

35. J.-T. Wu, L. He, M. X. Li and D. M. Lubman, J. Am. Soc. Mass Spectrom., in press. 